\title{
Das Forschungsnetzwerk Alterssicherung
}

Verstärkte Kooperation zwischen Rentenversicherung und Wissenschaft ${ }^{1}$

\section{Ausgangslage für eine verstärkte Kooperation zwischen Rentenversicherung und Wissenschaft}

Der Sozialstaat befindet sich in einer Phase grundlegender Veränderung. Die Gründe hierfür sind vielfältig. Die Industriestaaten müssen sich auf gravierende wirtschaftliche und gesellschaftliche Veränderungen einstellen. Stichworte sind die Globalisierung, d.h. die grenzüberschreitende Mobilität von Menschen und Kapital, verbunden mit einem immer stärker werdenden internationalen Wettbewerb der nationalen Wirtschaften und damit einhergehend auch der sozialen Sicherungssysteme, der Wandel hin zur Informationsgesellschaft, die Veränderung familiärer Strukturen, eine verstärkte Frauenerwerbstätigkeit, das Altern der Gesellschaften infolge niedrigerer Geburtenraten und längerer Lebenserwartung sowie der Trend $\mathrm{zu}$ Berufsverläufen mit häufigeren Wechseln. All das begründet erhebliche Anpassungszwänge in allen Systemen der sozialen Sicherung, insbesondere im Bereich der Alterssicherung.

Gerade Fragen der Alterssicherung und damit der gesetzlichen Rentenversicherung erfahren in den aktuellen Überlegungen zur Weiterentwicklung der sozialen Sicherungssysteme, nicht zuletzt aufgrund der hohen ökonomischen und sozialpolitischen Bedeutung dieses größten Einzelsystems sozialer Sicherung, große Beachtung: Ihre Anpassung an die sich verändernden gesellschaftlichen Rahmenbedingungen ist seit Jahren ein zentrales Anliegen staatlicher Sozialpolitik, verbunden mit einer entsprechenden öffentlichen und politischen Diskussion. Dieser Prozess ist weit vorangeschritten. Wurde vor der Reform 1992 auf der Basis des damals geltenden Rechts für 2030 ein Beitragssatz zur Rentenversicherung von 36 - 41 Prozent erwartet, so wird er heute mit der inzwischen beschlossenen Rentenreform 2004 auf 22,0 Prozent taxiert. ${ }^{2}$ Der größte Teil des Weges der notwendigen Reformen ist mit diesen Reformen bereits

1 Die vorliegenden Ausführungen basieren in Teilen auf: Ruland: Forschungsnetzwerk Alterssicherung - Verstärkte Kooperation zwischen Rentenversicherung und Wissenschaft im Bereich der Alterssicherung, DRV, Heft 3-4, 2001, S. 129 ff. Siehe allgemein zum Forschungsnetzwerk Alterssicherung: www.vdr-fna.de.

2 Siehe hierzu: Prognos: Gesamtwirtschaftliche Entwicklung und gesetzliche Rentenversicherung vor dem Hintergrund einer schrumpfenden Bevölkerung, Untersuchung im Auftrag des VDR, 1987, S. 59f. Vgl. zur Rentenreform 2004 u. a.: Reimann: Das RV-Nachhaltigkeitsgesetz - Gesamtwirkungen und Bewertung, DRV, Heft 6-7, 2004, S. 318 ff.; Ruland, Der neue Nachhaltigkeitsfaktor in der Rentenanpassungsformel und seine Auswirkungen, SGb 2004, Heft 6, S. 327 ff.; zu Einzelthemen: Hain / Lohmann / Lübke: Veränderungen bei der Rentenanpassung durch das »RV-Nachhaltigkeitsgesetz«, DRV, Heft 6-7, 2004, S. 333 ff.; Brall / Fasshauer / Lübke / Stahl: Neuregelungen im Bereich der Altersgrenzen, DRV, Heft 6-7, 2004, S. 350 ff.; Dünn / Lohmann / Stahl / Stegmann: Die Neuregelung zur Bewertung schulischer und beruflicher Ausbildungszeiten, DRV, Heft 6-7, 2004, S. 364 ff.; Stahl: Rentenrechtliche Änderungen bei Altersrenten, DRV, Heft 6-7, 2004, S. 384 ff. 
zurückgelegt. Trotzdem wird die Diskussion um die Zukunft der Alterssicherung und der Rentenversicherung weitergehen, zumal es nicht nur um sozialpolitische Zielsetzungen, sondern in hohem Maße auch um wirtschaftliche Interessen geht.

Der Rentenversicherung kommen in dieser Diskussion verschiedene Rollen zu. Im Wettbewerb der Systeme geht es um den ihr zugeordneten Aufgaben- und Verantwortungsbereich. Die Rentenversicherung kann in der aktuellen und künftigen Diskussion zur Zukunft der Alterssicherung in Deutschland mit guten Gründen für ihr System eintreten. Sie ist wegen ihrer in der Vergangenheit vielfach bewiesenen Anpassungsfähigkeit zukunftsfähig. Sie hat in ihrer über hundertjährigen Geschichte, zuletzt im Zuge der deutschen Wiedervereinigung, Herausforderungen in einer Weise gemeistert, zu der kein anderes System in der Lage gewesen wäre. ${ }^{3}$ Als gesetzliches Sicherungssystem bietet die Rentenversicherung zugleich eine gesellschaftspolitisch notwendige Solidarität, zu der kein privates System im Stande ist. ${ }^{4}$ All diese Vorzüge werden in der politischen, ebenso wie in der wissenschaftlichen Diskussion nicht immer hinreichend berücksichtigt. Die Rentenversicherung zugleich muss sich für ihr System auch deshalb einsetzen, weil es in dieser Auseinandersetzung vor allem um ihre Versicherten geht, die oft jahrzehntelang Beiträge entrichtet und damit eigentumsgeschützte Rentenanwartschaften erworben haben. ${ }^{5}$

Gerade aus der Verantwortung für ihre Versicherten ergibt sich für die Rentenversicherung aber auch die Aufgabe, für einen objektiven, sachangemessenen Diskurs der mit der Alterssicherung zusammenhängenden Fragen zu sorgen. Sie kann als mittelbar staatliche Institution diese Debatte nicht anderen Institutionen allein überlassen. Sie vor allem ist kraft ihrer Sachverantwortung auch für eine rationale, ergebnisoffene Diskussion der Fragen der Alterssicherung verantwortlich. Diese Verantwortung besteht gegenüber dem eigenen System: Soweit Anpassungsmaßnahmen zu ergreifen sind, müssen sie so frühzeitig wie möglich ergriffen werden, damit sich die Versicherten darauf einstellen können. Ende der 1970er Jahre war es die Rentenversicherung, die bereits zu diesem Zeitpunkt auf die Konsequenzen des Alterns unserer Bevölkerung für die Alterssicherung hingewiesen hat. ${ }^{6}$ Eine Verantwortlichkeit besteht aber

3 Siehe hierzu u. a.: Ruland: 100 Jahre Rentenversicherung - Bilanz eines sozialpolitischen Fortschritts, Sozialer Fortschritt 1991, S. 1 ff.; Ruland: Die Rentenversicherung in Deutschland im Zeichen der Jahrhundertwende, DRV, Heft 1-2, 2000, S. 23 ff.; Schmähl: Alterssicherung in Deutschland an der Jahrtausendwende - Konzeptionen, Maßnahmen und Wirkungen -, DRV, Heft 1-2, 2000, S. 50 ff.; Tennstedt: Geschichte des Sozialrechts, von Maydell/ Ruland (Hrsg.), Sozialrechtshandbuch (SRH), 3. Aufl., 2003, S. 24 ff.

4 Dazu ausf. Ruland, Solidarität und Individualität - ein allgemeines Thema konkretisiert am Beispiel der Rentenversicherung, DRV 2000, Heft 12, S. $733 \mathrm{ff}$.

5 Siehe zum Eigentumsschutz der Anwartschaften in der gesetzlichen Rentenversicherung u. a.: BVerfGE 53, 257 (289 ff.); 55, 114 (131); 58, 81 (109); 100, 1 (31); weitere Nachweise bei Ruland, Neugestaltung der Rentenformel, DAngVers 2000, Heft 5/6, S. 171 ff. Die Eigentumsgarantie erfüllt dabei für die durch sie geschützten rentenversicherungsrechtlichen Positionen gleichzeitig die Funktion des Vertrauensschutzes gegenüber Eingriffsakten, vgl. BVerfGE 45, 142 (168); 53, 257 (309); 58, 81 (120); 75, 78 (104 f.).

6 Kaltenbach, Bevölkerungsentwicklung und Rentenversicherung, DAngVers 1979, Heft 1, S. $1 \mathrm{ff}$. 
auch gegenüber der Politik, die über Änderungen des Rentenrechts zu entscheiden hat. Die Politik erwartet zu Recht Lösungsvorschläge der Rentenversicherung zu anstehenden Fragen. Sie sind stets unterbreitet worden; auch in der Diskussion um die nun beschlossene Rentenreform haben Lösungsvorschläge der Rentenversicherung, insbesondere des Verbandes Deutscher Rentenversicherungsträger (VDR), dazu beigetragen, dass die Reform gerechter wurde und die politischen Hürden nehmen konnte. ${ }^{7}$

Die Rentenversicherung und ihr Verband waren auch in der Vergangenheit stets um größtmögliche Rationalität bei der Erarbeitung ihrer Vorschläge und Meinungsäußerungen bemüht. Deshalb gibt es bereits seit vielen Jahren zahlreiche Querverbindungen zur Wissenschaft. Beispiele sind neben den Prognos-Gutachten, die der Verband in Auftrag gegeben hat, die von ihm initiierte und mittlerweile wiederholt durchgeführte Studie »Altersvorsorge in Deutschland « (AVID) ${ }^{8}$ und weitere Gutachten zu relevanten Fragestellungen (beispielsweise zu Verfassungsfragen der Organisationsreform). ${ }^{9}$ Auf Seiten des VDR sind vor allem seine Zeitschrift (»Deutsche Rentenversicherung [DRV]«), das von ihm mitherausgegebene »Handbuch der gesetzlichen Rentenversicherung « $(1990)^{10}$ sowie seine Schriftenreihe (»Schriften der DRV«) mit inzwischen 54 Bänden zu nennen. Hinzu kommen auch die zahlreichen vom VDR z.T. zusammen mit der Europäischen Rechtsakademie (ERA) und mit dem MaxPlanck-Institut für ausländisches und internationales Sozialrecht - veranstalteten Seminare.

Die anstehenden Fragen der Alterssicherung werden jedoch vielfältiger, zunehmend komplexer und sind daher immer schwieriger zu beantworten. Es bündeln sich in ihnen Aspekte aus den verschiedensten Wissenschaftsbereichen: Sozialrecht, Verfassungsrecht, Versicherungsrecht, Arbeitsrecht, Familienrecht, Europarecht, Sozialpolitik, Finanzpolitik, Gesellschaftspolitik, Volkswirtschaft, Statistik, Demografie, Soziologie, Medizin, Rehabilitationswissenschaft, Psychologie, Gerontologie, Politikwissenschaft, Versicherungswissenschaft und -mathematik, um nur die wichtigsten zu nennen. Selbst ein Verband, der auf die Interdisziplinarität seiner Mitarbeiter großen Wert legt, ist nicht mehr im Stande, all das Wissen vorzuhalten, das zur Beantwortung der vielgestaltigen Fragen notwendig ist, die sich im Zusammenhang mit der Alterssicherung stellen. Er muss, um seinen Aufgaben gerecht zu werden, in immer stärkerem Maße mit der Wissenschaft kooperieren, zumal er wegen seiner begrenzten Kapazitäten in personeller und finanzieller Hinsicht nur bedingt in der Lage ist, sich neben den

7 Vgl. u.a.: Ruland, Rolle und Bedeutung des Verbandes Deutscher Rentenversicherungsträger (VDR), DRV 1994, Heft 8, S. 543 ff.; VDR: Stellungnahmen zum Entwurf eines Gesetzes zur Sicherung der nachhaltigen Finanzierungsgrundlagen der gesetzlichen Rentenversicherung (»RV-Nachhaltigkeitsgesetz«), 2004.

8 Dazu Altersvorsorge in Deutschland 1996 - Lebensläufe und künftige Einkommen im Alter, DRV-Schriftenreihe Bd. 19 (= BMA Forschungsbericht Bd. 227), 1999.

9 Vgl. u. a.: Axer, Verfassungsrechtliche Fragen einer Organisationsreform in der Rentenversicherung, DRV-Schriftenreihe Bd. 24, 2000; Krebs, Verfassungsrechtliche Zulässigkeit der Organisationsreform der Deutschen Rentenversicherung, DRV-Schriftenreihe Bd. 14, 1999.

10 VDR/Ruland (Hrsg.), Handbuch der gesetzlichen Rentenversicherung, 1990. 
ihm zugedachten Koordinierungs- und Verwaltungsaufgaben mit den vielfältigen, neu aufkommenden Fragestellungen vertieft auseinander zu setzen.

Daher haben sich die Rentenversicherung und ihr Verband Ende der 90er Jahre eine verstärkte Kooperation zwischen Rentenversicherung und Wissenschaft im Bereich der Alterssicherung zum Ziel gesetzt, wie sie im Bereich der Rehabilitation bereits seit vielen Jahren erfolgreich praktiziert wird. ${ }^{11}$ Verschiedene Tagungen und Gespräche, insbesondere mit Wissenschaftlern im Bereich der Alterssicherungsforschung, haben die Notwendigkeit einer solchen verstärkten Kooperation deutlich bestätigt.

\section{Ziele des Forschungsnetzwerks Alterssicherung}

Die Rentenversicherung will durch die verstärkte Kooperation mit der Wissenschaft ihre wissenschaftliche, daher zwangsläufig ergebnisoffene Beratung in nationalen Fragen wie auch zunehmend in Fragen mit europäischen Bezug verbessern und intensivieren. Dies immer auch mit dem Ziel, die eigene Argumentation kritisch zu überprüfen. Dabei geht es um die Analyse von Grundsatzfragen und von spezifischen aktuellen Fragen verschiedener Disziplinen, wobei die Defizite im Bereich der empirischen Forschung, etwa der Wirkungsforschung, beseitigt werden sollen. Es geht aber auch um das rechtzeitige Erkennen von Tendenzen und Entwicklungen in Politik und Wissenschaft auf nationaler und internationaler Ebene: Ziele sind weiterhin der Ausbau der nationalen und internationalen Kontakte, internationaler Vergleiche und des Erfahrungsaustauschs im Sinne eines Benchmarkings. Die verstärkte Kooperation soll schließlich eine Plattform für die wissenschaftliche Auseinandersetzung zwischen der gesetzlichen Rentenversicherung, der Politik und der Wissenschaft schaffen und den (interdisziplinären) Austausch der Wissenschaftler untereinander fördern.

Mittelbare Ziele einer solchen verstärkten Kooperation der Rentenversicherung mit der Wissenschaft sind der Ausbau der wissenschaftlichen Infrastruktur und die Förderung des wissenschaftlich ausgebildeten Nachwuchses, an dem die Rentenversicherung auch als Arbeitgeber interessiert ist. Ein Ergebnis der bisherigen Erfahrungen ist, dass auch die Wissenschaftsdisziplinen, die sich mit dem Thema Altern und Alterssicherung beschäftigen, an der Kooperation mit der Rentenversicherung sehr interessiert sind. Sie brauchen für ihre Forschungen Fakten, Daten und Hintergrundwissen, das ihnen nur die gesetzliche Rentenversicherung bieten kann. Deshalb ist der VDR derzeit dabei, mit Unterstützung des Bundesforschungsministeriums ein Forschungsdatenzentrum aufzubauen. Die an Rentenfragen interessierte Forschung benötigt aber auch institutionelle Unterstützung, da ihr Standort und ihr Gewicht in der hochschulpolitischen Landschaft immer prekärer werden. Insbesondere Sozialrecht und Sozialpolitik besitzen in der universitären Ausbildung und Forschung nicht (mehr) den

11 Das Forschungsnetzwerk Alterssicherung betrifft damit nicht den Bereich der Rehabilitation. In ihm kooperieren die Rentenversicherung und ihr Verband schon seit vielen Jahren mit der Wissenschaft und finanzieren derzeit zusammen mit dem Bundesforschungsministerium den längerfristig angelegten Förderschwerpunkt Reha-Wissenschaften mit einem Fördervolumen von insgesamt 40 Millionen Euro. 
notwendigen Stellenwert, der einem Politikfeld mit hoher gesellschaftlicher und ökonomischer Relevanz, wie es die soziale Sicherung darstellt, zukommen müsste. Insofern ist die von der Rentenversicherung initiierte Kooperation eine Symbiose.

\subsection{Formen und bisherige Aktivitäten des Forschungsnetzwerks Alterssicherung}

Die intensive Kooperation mit der Wissenschaft kann sich nicht auf eine Fachrichtung beschränken, sie muss entsprechend der Komplexität der Fragestellungen interdisziplinär ausgestaltet sein. Es geht im Bereich der Alterssicherung zwar vornehmlich um sozialrechtliche und/oder sozialpolitische Fragen, diese müssen aber im Kontext mit volkswirtschaftlichen, politologischen, finanzwissenschaftlichen, soziologischen, psychologischen, mathematischen und medizinischen Fragestellungen gesehen und beantwortet werden. Daher zeichnete sich bereits zu Beginn des Forschungsnetzwerks Alterssicherung ab, dass unter den aufgeführten Zielen und der notwendigen interdisziplinären Ausrichtung im Wesentlichen nur die Bildung eines interdisziplinären wissenschaftlichen Netzwerkes als Kern der Kooperation den geeigneten organisatorischen Rahmen geben kann. Auch deshalb wurden Überlegungen in Richtung einer eigenen Forschungsabteilung innerhalb des Verbandes, eines Stiftungslehrstuhls oder einer Stiftungsprofessur an einer Universität sowie die Gründung eines An-Institutes bei einer wissenschaftlichen Einrichtung sehr rasch verworfen. Das Ziel war daher, ein »Forschungsnetzwerk « aus Wissenschaftlern zu gründen, die sich mit den die Rentenversicherung interessierenden Fragen und Themen beschäftigen. Dieses Netzwerk ist Informations- und Kooperationsbasis zugleich: Kooperation mit uns, der Rentenversicherung, aber auch der Wissenschaftler untereinander.

Die Rentenversicherung stellt damit die Infrastruktur - z. B. in Form der Datenbank mit mittlerweile mehr als 150 Wissenschaftlern - auf die die Beteiligten Zugriff haben. Unsere Zeitschrift »Deutsche Rentenversicherung (DRV)« steht diesem Netzwerk ebenso zur Verfügung, wie die Schriftenreihe (DRV-Schriftenreihe). In der Schriftenreihe sind bereits verschiedene, vom Forschungsnetzwerk Alterssicherung geförderte Projektberichte erschienen. ${ }^{12}$ Daneben sind in der DRV-Schriftenreihe und in der DRV auch diverse Tagungsberichte bzw. Tagungsbände von FNA-Veranstaltungen publiziert worden. ${ }^{13}$

Mit dem Forschungsnetzwerk Alterssicherung soll ebenso der wissenschaftliche Nachwuchs gefördert werden. Auch jüngere Wissenschaftler sollen die Chance bekommen, sich mit entsprechenden Arbeiten in dieses Themenfeld einzuarbeiten und

12 Folgende Projektberichte wurden bisher in der DRV-Schriftenreihe publiziert: Prognos AG: »Auswirkungen veränderter Rahmenbedingungen auf die Finanzen der gesetzlichen Rentenversicherung «, in: DRV-Schriftenreihe, 2004; Hase: »Steuerfinanzierte Beitragszuschüsse und Familienlastenausgleich «; in: DRV-Schriftenreihe, 2004.

13 Vgl. u.a.: VDR (Hrsg.): Besteuerung von Beiträgen und Leistungen in der Altersvorsorge, in: DRV-Schriftenreihe, 2001; VDR (Hrsg.): FNA-Jahrestagung 2001: »Offene Koordinierung der Alterssicherung in der Europäischen Union«, DRV-Schriftenreihe, 2002; VDR (Hrsg.): FNA-Jahrestagung 2002: »Arbeitsmarkt und Alterssicherung «, DRV-Schriftenreihe, 2003 und VDR (Hrsg.): FNA-Jahrestagung 2003: »Generationengerechtigkeit Inhalt, Bedeutung und Konsequenzen für die Alterssicherung«, DRV-Schriftenreihe, 2004. 
zu profilieren. Die Nachwuchsförderung erfolgt hierbei zum einen durch Stipendien, zum anderen durch ein jährlich stattfindendes Graduiertenkolloquium und zum Dritten durch den jährlich zu vergebenden FNA-Forschungspreis. ${ }^{14}$

Die Kooperationsformen zwischen Wissenschaft und Rentenversicherung lassen sich damit in fünf Bereiche untergliedern:

- Förderung von Projekten: Das Forschungsnetzwerk fördert Projekte zu grundsätzlichen Fragestellungen ebenso wie Forschungsvorhaben zu aktuellen rentenpolitischen Überlegungen.

- FNA-Forschungspreis: Der jährlich zu vergebende FNA-Forschungspreis dient der Anerkennung weit überdurchschnittlicher Leistungen junger Wissenschaftler (vor allem Dissertationen) auf dem Gebiet der Alterssicherung.

- Tagungen: Für die Kommunikation zwischen Wissenschaft und Praxis werden vom FNA regelmäßig Tagungen durchgeführt. Die Veranstaltungen umfassen neben der jährlich stattfindenden FNA-Jahrestagung auch Workshops, Symposien und Graduiertenkolloquien.

- Datenbank: Das FNA nutzt die modernen Kommunikationsmöglichkeiten und hat eine umfangreiche Datenbank aufgebaut.

- Stipendien: Das FNA unterstützt die universitäre Grundlagenforschung und bietet qualifizierten Nachwuchswissenschaftlern die Möglichkeit, Forschungsvorhaben zu Fragen der Alterssicherung, die zugleich der Erlangung eines akademischen Grades dienen, mittels eines Stipendiums zu finanzieren.

Als Beratungsforum für Forschungsvorhaben und zur Sicherung der wissenschaftlichen Qualität der Forschungsmaßnahmen wurde ein interdisziplinär ausgerichteter Beirat eingesetzt. Er setzt sich aus 16 Personen zusammen: acht Vertreter der Wissenschaft und acht Vertreter der Rentenversicherung. ${ }^{15}$ Die zentralen Aufgaben des Beirats bestehen in der Unterstützung der Rentenversicherung bei der Wahl der Forschungsschwerpunkte, beim Aussprechen von Empfehlungen über die Durchführung von Projekten sowie bei der Evaluierung der im Rahmen der verstärkten Wissenschaftskooperation durchgeführten Forschungsvorhaben.

\section{Thematische Schwerpunktsetzung des Forschungsnetzwerks Alterssicherung}

Bereits bei den Vorarbeiten zur Initiierung des Forschungsnetzwerks Alterssicherung zeigte sich eine weitgehende Übereinstimmung zwischen dem innerhalb der Rentenversicherung gesehenen Forschungsbedarf und den von der Wissenschaft festgestell-

14 Der FNA-Forschungspreis 2002 wurde an Frau Dr. Hamisch für ihre Dissertation »Der Schutz individueller Rechte bei Rentenreformen - Bundesrepublik Deutschland und Großbritannien im Vergleich« und 2003 an Herrn Prof. Dr. Wagener für seine Habilitationsschrift: »Old-Age-Provisions under Uncertainty« vergeben.

15 Mitglieder des FNA-Beirats sind: Dr. Hans J. Barth, Prof. Dr. Ulrich Becker, Manfred Burmeister, Prof. Dr. Dr. h.c. Eberhard Eichenhofer, Alexander Gunkel, Prof. Dr. Klaus Heubeck, Dr. Harald Huhn, Prof. Dr. Rainer Münz, Dr. Axel Reimann, Prof. Dr. Barbara Riedmüller, Dr. Herbert Rische, Prof. Dr. Dr. h.c. Bert Rürup, Prof. Dr. Franz Ruland, Günter Schäfer, Prof. Dr. Winfried Schmähl und Dr. Erich Standfest. 
ten Forschungsdefiziten im Bereich der Alterssicherung. In den vergangenen Jahren wurden eine Vielzahl forschungsrelevanter Themen vorgetragen, die sich weitestgehend auf (mittlerweile) fünf Themenkomplexe konzentrierten:

- Ziele der Alterssicherung,

- Wirkungsanalyse des Alterssicherungssystems,

- Akzeptanz des Alterssicherungssystems,

- Europäische und internationale Alterssicherungspolitik und

- Sicherung bei Erwerbsminderung.

Dabei bestehen zwischen einzelnen Themen aus den verschiedenen Bereichen eine Vielzahl von Anknüpfungspunkten.

Gerade im Zuge der Auseinandersetzungen über die kürzlich beschlossene Rentenreform 2004 hat sich wiederholt gezeigt, dass die Gestaltung der Alterssicherung einer Untersuchung der »Ziele der Alterssicherung « bedarf: ${ }^{16}$ Welche Sicherung sollte im Alter bestehen, wie sollte sie zusammengesetzt sein, welche Sicherung kann und muss der Staat über die gesetzliche Rentenversicherung gewährleisten? Dies setzt auch eine bessere Kenntnis über den tatsächlichen Sicherungsbedarf und über die tatsächlich erreichten Sicherungsniveaus voraus - eine ebenfalls noch zu untersuchende Forschungsfrage. Dabei können die Ziele der Alterssicherung nicht allein auf die Dimension der Einkommenssicherung begrenzt werden, sondern sie müssen auch die weiteren Dimensionen des Alterns umfassen - beispielsweise Bedarfslagen, die Leistungen erfordern, die z. T. nicht über den Markt abgedeckt werden können. Notwendiger Forschungsbedarf findet sich auch in Bezug auf die Höhe des Einkommens, welches nach dem Übergang von der Erwerbsphase in die Rente ein annähernd gleiches Wohlstandsniveau gewährleisten soll, wie zuvor das Erwerbseinkommen. Bei einer solchen Bestimmung der Einkommenssituation im Alter sind auch die akkumulierten Vermögen zu berücksichtigen. In diesen Komplex fallen des Weiteren auch Fragen der Besteuerung der Alterssicherung.

Bei der Diskussion der Ziele muss auch die Frage nach inter- und intragenerativer Gerechtigkeit gestellt werden. Dabei ist zu beachten, dass die Frage nach der Gerechtigkeit zwischen den Generationen nicht auf die Betrachtung der Alterssicherung beschränkt werden darf, wie sich auf der FNA-Jahrestagung 2003 eindrucksvoll zeigte. ${ }^{17}$ In engem Zusammenhang hierzu finden sich Forschungsthemen, die sich mit der Zielerreichung unter den gegeben Restriktionen der Mittelknappheit und der demografischen Entwicklung beschäftigen. Die bisher genannten Themen umfassen die Ausgestaltung der verschiedenen Säulen der Alterssicherung ebenso wie das Entscheidungsverhalten der Bürger sowie die Zuwanderung. Bei dem letzteren Punkt müssen beispielsweise auch der Zuwanderungsbedarf und die Möglichkeiten, ihn zu decken, thematisiert werden.

16 Derzeit fördert das FNA ein zweijähriges Projekt mit dem Titel: »Zielvorstellungen in der Alterssicherung: Konkretisierung, Operationalisierung und Messung als Grundlagen für normative Aussagen «. Weitere Informationen hierzu finden sich unter: www.vdr-fna.de.

17 Vgl. hierzu: VDR (Hrsg.): FNA-Jahrestagung 2003: »Generationengerechtigkeit - Inhalt, Bedeutung und Konsequenzen für die Alterssicherung«, DRV-Schriftenreihe, 2004. 
Bei der Betrachtung der Ziele der Alterssicherung und des Grades der Zielerreichung gilt das Erkenntnisinteresse der Rentenversicherung und der Wissenschaft in besonderem Maße den Beschränkungen beim Aufbau einer angemessenen Altersvorsorge. Diese umfassen zum einen Fragen des durchgehenden oder unterbrochenen Erwerbsverlaufs und seiner Bedeutung für die Alterssicherung. Zum anderen gilt das Erkenntnisinteresse der Stetigkeit der Versorgung im Alter, mit vornehmlichem Blick auf die gestiegene und weiter steigende Lebenserwartung. Weitere Forschungskomplexe betreffen die Problematik des notwendigen Anstiegs der Lebensarbeitszeit sowie die Verteilungswirkungen der finanziellen Folgen einer steigenden Lebenserwartung. Forschungsbedarf zeigt sich ferner bei den Zugangsmöglichkeiten zu den Alterssicherungssystemen sowie bei der Frage, welche Bevölkerungsgruppen in welcher Weise in die Solidarität mit einbezogen werden sollen.

Den zweiten Forschungsschwerpunkt stellen »Untersuchungen der Wirkungen« dar, die sowohl durch angestrebte als auch durch nicht-intendierte Anreize des Alterssicherungssystems ausgelöst werden. ${ }^{18}$ Hierunter fallen Forschungsfragen, die sich mit den Wirkungen auf das generative Verhalten, dem Einfluss einer Verschiebung des Wohlfahrtsmixes, den Auswirkungen auf das Arbeitsangebot und die Humankapitalbildung, der Risikobereitschaft und den Reaktionen auf institutionelle Änderungen in anderen Bereichen der Wirtschaft und der Gesellschaft beschäftigen. Hier stellt sich auch die Frage nach den Wirkungen des Alterssicherungssystems auf Beschäftigung und Investitionsverhalten. Auch die Forschungsfrage nach dem Verhalten der Bürger in komplexen Entscheidungssituationen ist bei der Untersuchung von Wirkungszusammenhängen neu zu stellen. Ebenfalls diesem Themenkreis zuzurechnen ist die Forderung nach einer möglichst zeitnahen Untersuchung der Wirkungen der vergangenen Rentenreformen.

Im Zuge der Diskussionen innerhalb der Rentenversicherung, aber auch zwischen Rentenversicherung und Wissenschaft haben sich Fragestellungen um den Begriff »Akzeptanz" als weiterer Forschungsschwerpunkt herausgebildet, der ebenfalls in Ansätzen Eingang in die FNA-Forschungsaktivitäten gefunden hat. ${ }^{19}$ Hierbei geht es in erster Linie um Forschungsfragen, die sich mit der Wahrnehmung des Alterssicherungssystems durch die Bürger beschäftigen. Dies umfasst beispielsweise Wahrnehmungen differenziert nach dem Informationsstand der Bürger oder nach Alterskohorten. Die dem Denken der Bürger zu Grunde liegenden normativen Modelle sollten im Zusammenhang mit der jeweiligen Erwartungsbildung untersucht werden. Auch besteht Erkenntnisinteresse gegenüber den Einstellungen zu den abgesicherten Risiken und gegenüber der sich daraus ableitenden Legitimität von Ansprüchen. Diese Fragen

18 Das FNA fördert in diesem Themenschwerpunkt zur Zeit insbesondere in Form der Erstellung einer auf zwei Jahre angelegten Projektarbeit zum Thema »Dynamisches Analysesystem Alterssicherung: Simulationsbasierte Wirkungsanalyse der Alterssicherung im MehrSäulen-System «. Auch hierzu finden sich weitere Informationen unter: www.vdr-fna.de.

19 Siehe hierzu u. a.: FNA-Workshop: »Wissen über die Rentenversicherung und ihre Beurteilung im Wertewandel«, 4. / 5. Juli 2002 mit diversen Beiträgen in: DRV 2002, Heft 9-10, S. 473 - 538; sowie Inifes: »Zusammenstellung von Umfrageergebnissen zu Akzeptanz von und Wissen über die gesetzliche Rentenversicherung«, 2003 (FNA-Projektbericht); s. a. Heien, Das Vertrauen der Bürger in die Rentenversicherung, DRV 2004, Heft 4, S. 211 ff. 
stellen sich z.B. im Kontext der Hinterbliebenensicherung mit besonderer Dringlichkeit, da sie zu Gunsten einer Individualisierung der Ansprüche zunehmend in Frage gestellt wird.

Mit »Europäische und internationale Alterssicherungspolitik« ist der vierte Forschungsschwerpunkt umschrieben. Es bestand und besteht in Teilen noch ein erheblicher Forschungsbedarf bei der Untersuchung des Einflusses des europäischen Integrationsprozesses und der supranationalen Verflechtungen auf die nationalen Alterssicherungssysteme. Dies betrifft den unmittelbaren Einfluss von Rechtsetzung und Rechtsprechung auf der EU-Ebene, aber auch von »soft law « (Kommissionsmitteilungen, internationale Vereinbarungen etc.) auf die nationale Rechtsentwicklung. Zudem geht es um die mittelbaren Folgewirkungen, die sich aus dem Zusammenwachsen zu einem Binnenmarkt und aus der Erweiterung der EU für die nationale Sozialpolitik ergeben. Ein besonderes Interesse gilt dabei dem von der EU-Kommission entwickelten Verfahren der »offenen Koordinierung « und der darin vorgesehenen Entwicklung von sozialen Indikatoren, die als Leitlinien und Bewertungsmaßstäbe für die nationale Sozialpolitik dienen. Dabei geht es grundsätzlich darum, ob und inwieweit die Systemkonkurrenz innerhalb Europas zu einem Sozialdumping, d. h. zu einem Wettbewerb »nach unten«, oder zur Evolution eines »europäischen Sozialmodells« führen wird. Die damit verbundene Einengung des zukünftigen nationalen Gestaltungsspielraums der Alterssicherungspolitik berührt damit auch den Forschungsschwerpunkt »Ziele der Alterssicherungssysteme«. Außerdem besteht bei dem Anliegen, die Wirkungen nationaler Entscheidungen im Bereich der Alterssicherung auf das europäische Umfeld zu untersuchen, ein direkter Zusammenhang zum zweiten Forschungsschwerpunkt (»Wirkungsanalyse des Alterssicherungssystems«).

Das Forschungsnetzwerk Alterssicherung hat diesen Aspekt des Forschungsschwerpunkts »Europäische und internationale Alterssicherungspolitik « bereits frühzeitig aufgegriffen und in unterschiedlicher Weise zu beleuchten versucht. So wurden u. a. zwei internationale Tagungen in Kooperation mit dem Bundesministerium für Arbeit bzw. dem Bundesministerium für Gesundheit und Soziale Sicherung und dem MaxPlanck-Institut für ausländisches und internationales Sozialrecht durchgeführt und Tagungsbände hierzu in Deutsch, Englisch und Französisch publiziert. ${ }^{20}$ Die Rentenversicherung hat insbesondere mit diesen Tagungen die Möglichkeit des Austausches zwischen Wissenschaft und Praxis eröffnet und damit aktiv den Weg in diesem Bereich in den vergangenen Jahren mitgestaltet.

Einen weiteren wichtigen Schwerpunkt im Rahmen des Forschungsschwerpunkts »Europäische und internationale Alterssicherungspolitik« stellen die EU-Osterweiterung und ihre Konsequenzen dar. So fehlten bis vor wenigen Jahren systematische Untersuchungen über die Alterssicherungssysteme in den Beitrittsstaaten, die es aber mittlerweile in zunehmenden Maße gibt. Zu klären sind aber noch die Auswirkungen

20 Vgl. VDR (Hrsg.): FNA-Jahrestagung 2001: »Offene Koordinierung der Alterssicherung in der Europäischen Union«, DRV-Schriftenreihe Bd. 34, 2002; VDR (Hrsg.): »Offene Methode der Koordinierung im Bereich Alterssicherung - Quo Vadis?«, DRV-Schriftenreihe Bd. 47, 2003. 
des Beitritts der neuen EU-Mitglieder auf das bundesdeutsche und die anderen europäischen Systeme der Alterssicherung. Zudem ist es notwendig, Umfang und Wirkungen der kurz- bis mittelfristigen Migration auch im Gefolge der EU-Osterweiterung zu analysieren. In diesem Zusammenhang wurde von Vertretern der Wissenschaft hervorgehoben, dass zwar von der EU Mittel für europaspezifische und vergleichende Forschung bereit gestellt werden, dass diese jedoch nicht für deutschlandspezifische Fragestellungen zur Verfügung stehen. Gerade für derartige Fragestellungen wurde jedoch von den Beteiligten in der Rentenversicherung und in der Wissenschaft ein besonderer Forschungsbedarf konstatiert.

In den vergangenen Jahren hat sich herausgestellt, dass ein weiterer, fünfter Bereich mit dem Titel »Sicherung bei Erwerbsminderung « eingeführt werden sollte. Das Thema hat nach Einschätzung der Rentenversicherung, aber auch der Wissenschaft in der Forschung bisher keine der Bedeutung entsprechende Berücksichtigung gefunden. $\mathrm{Zu}$ untersuchende Fragestellungen beziehen sich beispielweise auf die Auswirkungen der grundlegenden Reformmaßnahmen im Bereich der Erwerbsminderung im Jahre 2001 oder aber auch auf die Bedeutung der Absicherung der Erwerbsminderung bei einer Veränderung der Altersgrenzen.

\section{Stand der Umsetzung}

Wie die Ausführungen zu den einzelnen Formen der verstärkten Kooperation zwischen Rentenversicherung und Wissenschaft, der organisatorischen Ausgestaltung sowie den thematischen Schwerpunkten gezeigt haben, sind bereits wichtige Schritte für eine verstärkte Kooperation zwischen Rentenversicherung und Wissenschaft im Rahmen des Forschungsnetzwerks Alterssicherung zurückgelegt worden. Basierend auf der in den vergangenen Jahren aufgebauten Infrastruktur, stehen die weitere, konkrete Umsetzung der inhaltlichen Ziele durch die Förderung von Forschungsprojekten, der Aufbau des Forschungsdatenzentrums, die Vergabe von Stipendien, die Organisation von Tagungen und Graduiertenkolloquien usw. im Mittelpunkt der FNA - Aktivitäten.

Hervorzuheben ist die große Unterstützung innerhalb der Rentenversicherung und im Bereich der Wissenschaft für die Idee des Forschungsnetzwerkes Alterssicherung von Beginn an. So haben sich parallel zu den Arbeiten des Verbandes alle zuständigen Gremien der Rentenversicherung mit dem Thema »verstärkte Kooperation zwischen Rentenversicherung und Wissenschaft « beschäftigt und das vorgestellte Konzept begrüßt und ausdrücklich bestätigt. Auf der Mitgliederversammlung im Oktober 2000 wurden daher erstmals für das Jahr 2001 Forschungsmittel in Höhe von 1,5 Mio. DM im Haushalt des Verbandes angesetzt; dieser Betrag ist im Zuge der Euroumstellung inzwischen auf 750.000 Euro p. a. festgelegt worden. Diese finanziellen Mittel gilt es unter den bereits genannten hohen Anforderungen für die vorgesehenen Forschungsmaßnahmen in entsprechender Weise einzusetzen. 


\section{Ausblick}

Die Ausführungen haben deutlich gemacht, dass eine enge Kooperation zwischen Wissenschaft und Rentenversicherung notwendig ist. Die gesetzliche Rentenversicherung ist bereit, mit diesem bisher erfolgreich verlaufenen Projekt weiter auf die Wissenschaft zuzugehen. Die Resonanz aus der Wissenschaft ist bisher sehr erfreulich. Auch künftig bleibt die Rentenversicherung - auch in der Organisation des Netzwerks - auf die Mithilfe der Wissenschaft angewiesen.

Eine vertiefte Kooperation zwischen Rentenversicherung und Wissenschaft wird zu einer sachgerechteren Diskussion der mit der Alterssicherung zusammenhängenden komplexen Fragen und zu rationalen politischen Entscheidungen führen. Damit ist die verstärkte Kooperation zwischen Rentenversicherung und Wissenschaft nicht zuletzt auch eine sachgerechte Investition in die Zukunft im Interesse der Beitragszahler.

Als eine auch politische Anerkennung dieser Forschungsinitiative des VDR ist es zu werten, dass die »Forschung im Bereich der Alterssicherung und der Rehabilitation« zu dem gesetzlichen Aufgabenbereich der neuen Spitzeninstitution der Rentenversicherung gehören soll, die sich im Zuge der geplanten Organisationsreform der Rentenversicherung aus dem Zusammenschluss von VDR und der Bundesversicherungsanstalt für Angestellte (BfA) ergeben soll. ${ }^{21}$

21 Vgl. § 138 Abs.1 Nr. 15 SGB VI i.d.F. des Entwurfs eines Gesetzes zur Organisationsreform in der gesetzlichen Rentenversicherung (RVOrgG), BR-Dr. 430/04. 